\title{
Article \\ Effectiveness of an App-Based Mobile Intervention for Precision Oral Self-Care in Patients with Periodontitis from Initial Therapy to Re-Evaluation
}

\author{
Wen-Jen Chang ${ }^{1,2, *}$, Yen-Li Wang ${ }^{3}$, Yen-Hsiang Chang ${ }^{2}$ and Shih-Yin Lo ${ }^{3}$ \\ 1 Department of Information Management, Chang Gung University, Taoyuan 33302, Taiwan \\ 2 Department of Dentistry, Chang Gung Memorial Hospital, Taoyuan 33302, Taiwan; cyh4714@hotmail.com \\ 3 Department of Periodontics, Chang Gung Memorial Hospital, Taoyuan 33302, Taiwan; \\ wanglacy519@gmail.com or m5975@cgmh.org.tw (Y.-L.W.); onsale42@cgmh.org.tw (S.-Y.L.) \\ * Correspondence: wjchang@gap.cgu.edu.tw
}

Citation: Chang, W.-J.; Wang, Y.-L.; Chang, Y.-H.; Lo, S.-Y. Effectiveness of an App-Based Mobile Intervention for Precision Oral Self-Care in Patients with Periodontitis from Initial Therapy to Re-Evaluation. Appl. Sci. 2021, 11, 4229. https:// doi.org/10.3390/app11094229

Academic Editor: Paola Gandini

Received: 1 April 2021

Accepted: 4 May 2021

Published: 7 May 2021

Publisher's Note: MDPI stays neutral with regard to jurisdictional claims in published maps and institutional affiliations.

Copyright: () 2021 by the authors. Licensee MDPI, Basel, Switzerland. This article is an open access article distributed under the terms and conditions of the Creative Commons Attribution (CC BY) license (https:// creativecommons.org/licenses/by/ $4.0 /)$.

\begin{abstract}
Good oral hygiene is essential to oral health and oral disease prevention. Strengthening the adherence of patients with periodontitis to oral hygiene behaviors (OHBs) is crucial for the success of periodontal treatment. This study aims to investigate the intervention effectiveness of a novel mobile app for precision oral self-care, which was developed to help patients with periodontitis improving their oral hygiene through daily oral self-care. One-hundred and fifty eligible patients were recruited into this study and randomly allocated to the control $(n=75)$ or intervention group $(n=75)$, and their oral hygiene status (OHS) and OHBs were recorded at baseline and re-evaluation. The participants in the intervention group were required to perform daily oral self-care using our oral self-care app (OSCA) and the functional preferences and overall likeability of the OSCA were evaluated through a questionnaire survey. In total, 39 patients from the control group and 49 from the intervention group completed clinical examinations at baseline and re-evaluation. The results demonstrated that there was no significant difference in OHS improvement between the two groups. However, OHS improvement significantly differed between low-, moderate-, and high-frequency users, with frequency of use being positively correlated to the likeability of the app. Further investigation of the effectiveness of the OSCA intervention revealed that behavioral change in oral hygiene, frequency of OSCA use, and smoking affected OHS improvement. These outcomes indicate that our app-based mobile intervention may be of value for precision oral self-care in patients with periodontitis.
\end{abstract}

Keywords: oral hygiene; periodontitis; oral health promotion; mobile health; behavior change techniques; behavior intervention

\section{Introduction}

Oral health is highly related to one's general health, well-being and quality of life [1]. Good oral hygiene protects an individual's oral health and serves as a foundation of overall health, whereas poor oral hygiene leads to such dental problems as tooth decay and periodontal diseases, which in turn affect daily life. Maintaining good oral hygiene can sustain chewing ability and prevent bad breath, thereby enhancing personal social interactions, self-confidence, and happiness [2]. However, the prevalence of oral diseases is extremely high. Almost $100 \%$ of adults and $60 \%-90 \%$ of schoolchildren worldwide suffer from dental caries [3]. Periodontal disease is a common dental disease in adults, with a global prevalence rate of $20 \%-50 \%$, and severe periodontitis is the sixth most prevalent oral disease worldwide [4].

Good behavior in oral self-care is fundamental to maintaining one's oral health and an important personal responsibility [5]. It is well known that daily oral self-care can effectively improve oral health and prevent dental or periodontal diseases. However, a significant number of individuals still suffer greatly from oral diseases, primarily owing 
to incorrect or inadequate knowledge of and inappropriate skills in oral care [5]. Providing oral care knowledge and skills is thus necessary to establish good behavior and capability to enhance oral hygiene $[5,6]$. Dental practitioners provide patients with oral hygiene instructions (OHIs) according to the results of examination during dental visits; however, poor adherence to these instructions is one of the key factors that negatively affect the success of dental treatments $[7,8]$. The most common barrier to proper adherence to OHIs is memory burden, which refers to patients' difficulty with recalling much of the information that they had received $[9,10]$. Practical functions of interventions could significantly reduce this burden in patients with periodontal diseases and improve their adherence to OHIs $[10,11]$.

The past decade has witnessed the popularization and ubiquity of mobile apps, which have been identified as useful intervention tools for improving the efficiency of individuals' self-care and speed of healthcare delivery $[5,6,10,12]$. The potential of appbased interventions in promoting health and well-being is ever increasing [13], and the number of health-related apps aiming to promote health behaviors has grown substantially as a result. More than 325,000 mobile health apps were available for download worldwide in 2017 [14], with an exponential growth [15]. However, the extent to which the content of these apps can facilitate behavioral change varies. Therefore, it is important to identify the factors that allow a health promotion app to successfully support changes in health behavior [13].

Behavioral change techniques are theory-based methods that have been reported to help individuals change their behaviors to achieve better health $[12,16]$ and are commonly used as intervention mechanisms [16-20]. Studies have reported that health promotion apps based on behavioral change techniques are more likely to be effective in eliciting changes in behaviors $[11,13,17,18]$. However, many of the current apps were developed without adopting such techniques [12]. Lack of expert participation in the design stage, evidence, and a theoretical base to support interventions has been cited as the main reason for poor uptake and discontinued use of mobile health apps [13,17]. Apps designed with content that includes the opinions of clinicians, interventions developed in evidence-based practice, and internal drivers, such as motivations, tend to be more effective in facilitating behavioral change compared with those that were not $[13,18]$.

The number of oral health promotion apps is much lower than that of other healthrelated apps, especially for adults. Most of the existing oral care apps were developed without the participation of oral health experts and with information that has not been validated [19-21]. The evidence base supporting the use of apps is scant, and patients' perspectives are seldom taken into account during the design stage [13,21]. Furthermore, the lack of professional regulation to approve developed apps indicates that the information contained in apps could be ambiguous and unreliable. Reviews of oral health apps have concluded that the quality of apps is generally poor and pointed out that the development of oral care apps to promote oral hygiene is still in its infancy $[12,21,22]$.

The objective of this study was to evaluate the intervention effectiveness of the oral self-care app (OSCA) [10], which was developed based on the framework of the Behavior Change Wheel (BCW) [23]. The effectiveness of the intervention was assessed by the difference in patients' oral hygiene status (OHS) between baseline and re-evaluation through clinical examinations.

\section{Materials and Methods}

We conducted a pre- and post-intervention study on patients with periodontitis to evaluate the effectiveness of the OSCA intervention. Ethical approval was obtained from the institutional review board of Chang Gung Memorial Hospital (Taoyuan, Taiwan) (Approval No. 201701853B0). Participants were divided into two groups, namely, a control group and intervention group, to compare the difference in the improvement of OHS between use of traditional OHIs and intervention with the OSCA. In the control group, participants received advice and OHIs from dental care team after their clinical examination 
and then performed daily oral self-care accordingly. On the other hand, participants in the intervention group not only received the same advice and OHIs as the control subjects did but also were requested to install the OSCA to assist them in performing daily oral self-care.

\subsection{Inclusion Criteria and Procedure}

Patients attended to the periodontal clinic of the medical center with a diagnosis of periodontitis and fulfilled the National Health Insurance of Taiwan requirements for periodontal initial therapy after clinical examination between Jane 2018 and March 2019 were eligible to participate in this study. The above-mentioned requirements included the following: diagnosis of a general aggressive or chronic periodontitis requiring nonsurgical treatment only, presence of at least 16 teeth, at least 6 of which with a probing depth of $5 \mathrm{~mm}$ or greater, and not having received treatment in a specialist clinic in the past year. One-hundred and fifty patients volunteered to take part in the study and provided written informed consent before their enrollment. A flowchart of the participants is shown in Figure 1.

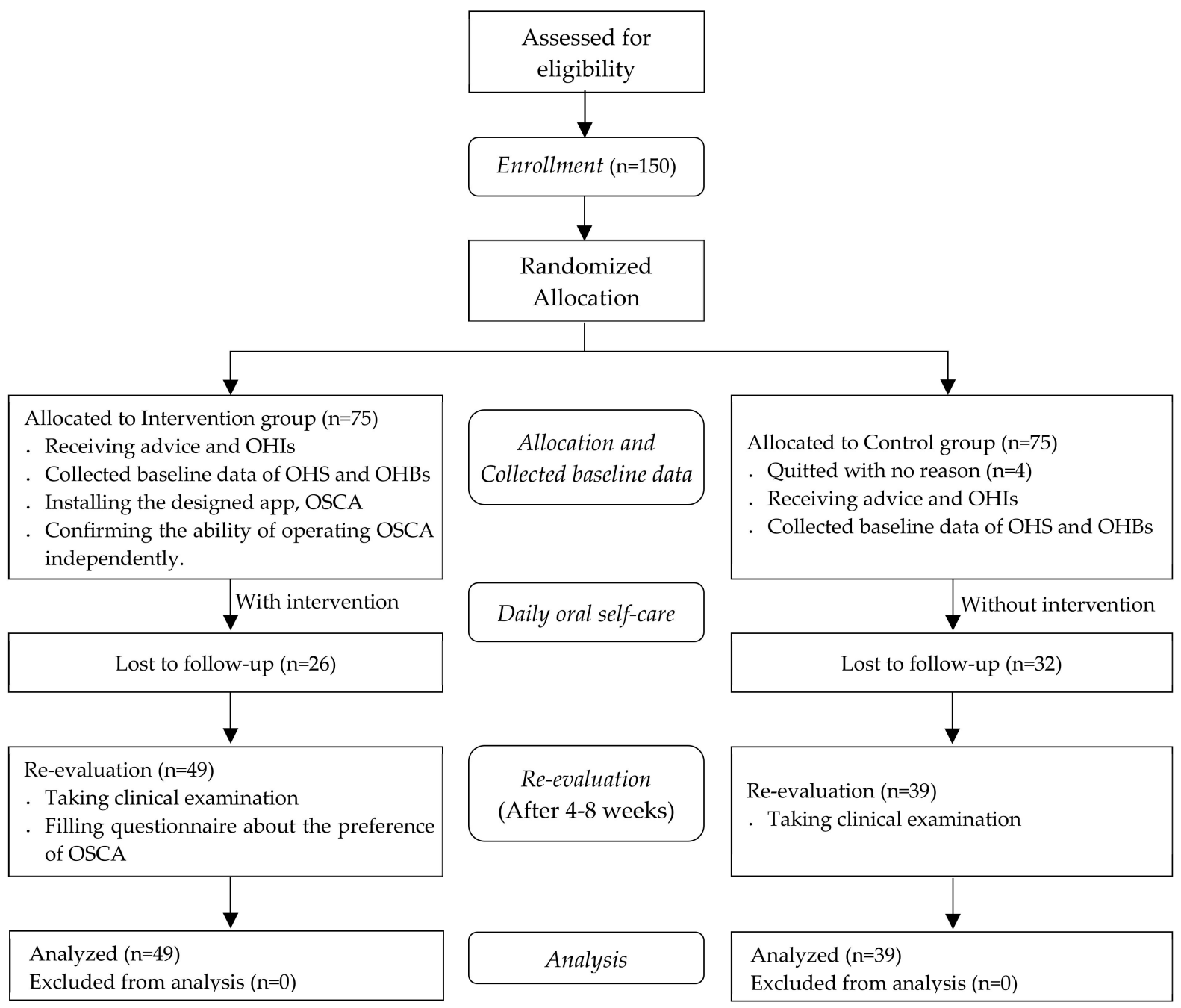

Figure 1. Flowchart of the study participants.

Participants were randomly allocated to the control or intervention group. The patients underwent clinical examination from which baseline data on their OHS and daily oral hygiene behaviors (OHBs) were collected. The patients were given advice and OHIs on oral self-care for improving their oral health status based on their clinical examination. 
Participants received periodontal initial therapy (including scaling and root planning) during the visit of the baseline and scheduled follow-up appointments for re-evaluation after their treatment. The appointments were scheduled between 4 to 8 weeks, which was proposed to be an ideal time for re-evaluation after periodontal initial therapy [24]. The participants in the intervention group were requested to install the OSCA on their smartphone to receive intervention for daily oral self-care until their re-evaluation visit. To provide personalized and tailored information, periodontist highlighted the focal areas that required extra cleaning effort in the dental chart on the app according to the results of the patients' clinical examination. A research assistant introduced the features of the OSCA in person and participants were asked to complete the specified tasks independently after practice to ensure their ability to operate the OSCA.

Participants revisited to the clinics for a re-evaluation of their oral health by the same periodontist, and the data on their OHS and OHBs were collected again. Participants in the intervention group were asked to complete a questionnaire that was designed to measure their overall likeability and functional preferences to the OSCA.

\subsection{Intervention}

OSCA used in this study was developed based on the BCW framework [23] and its usability was examined [10]. We considered how the app could be used to engage the participants and facilitate behavioral change in their oral self-care. Therefore, at the design stage, we invited clinical experts (two periodontists and one head nurse of the dental services department of our institution) to participate in the discussion and share their professional comprehensive knowledge on oral self-care for patients with periodontitis. The OSCA has two key features: evidence-based and personalized. The oral self-care knowledge and skills incorporated in the app were extracted from clinical practice guidelines, and personalized, tailored information was provided according to the results of each patient's clinical examination. Participants in the intervention group were asked to perform daily oral self-care using the OSCA for approximately $4-8$ weeks until their re-evaluation visit.

The functions of the OSCA were categorized into capability establishment, motivation enhancement, and opportunity creation (Figure 2). The function of capability establishment is the core value of the OSCA, the periodontists indicated that insufficient knowledge about and incorrect skills in oral self-care are the primary barriers to maintaining oral health in patients with periodontitis. Lack of appropriate information and the burden of recalling the OHIs received from the dental care team were reported as the main reasons for poor adherence to good oral self-care $[9,10]$. Therefore, establishing capability for these patients is fundamental to improving their OHS. The OSCA was designed to establish patients' capability by providing knowledge and personalized, tailored information in accordance with the findings of their clinical examination. Participants could view the information anytime and anywhere, which reduced the burden of recalling such important data. The motivational enhancement function was created to enhance the participants intention to engage in the target behavior of oral self-care, and the opportunity creation function was included to transform motivation into action, which makes behavioral change in performing good oral self-care possible [10]. Use of the OSCA was expected to result in behavioral change in long-term oral self-care for the participants.

\subsection{Measurements}

Demographic information (including gender, age, and education level) and smoking habit of the participants were collected when they enrolled in the study. Their daily OHBs, number of remaining teeth, and OHS were recorded at baseline and re-evaluation. Participants in the intervention group were asked to respond to questions about the functional preferences and overall likeability of the OSCA. 


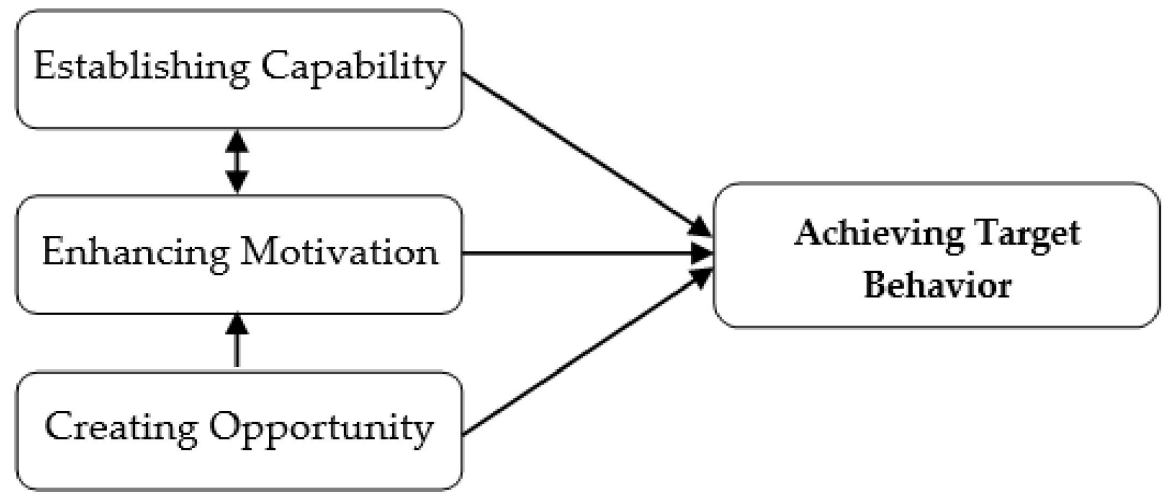

Figure 2. Conceptual design of the OSCA.

OHBs for daily self-care were measured by four items that were adapted from the instrument used by Brein et al. [25]. A composite variable was constructed with the sum of the values of these four items to assess the respondents' OHBs. The summary OHB score ranged from 4 to 12, with a high summary score indicating a high level of OHBs.

Plaque control level, which has been widely used to assess patients' ability to control plaque, was used as the OHS indicator in this study. The level of plaque control for each participant was evaluated using a modified version of the O'Leary Plaque Control Record (PCR) [26], which has been used as an oral hygiene index for assessing oral health skills since it was first introduced in 1972 [27]. According to the PCR, the presence or absence of supragingival plaque was recorded in six sites (disto-buccal, mid-buccal, mesio-buccal, disto-lingual, mid-lingual, and mesio-lingual) of each tooth after the disclosing solution was applied. PCR scores, which range from 0 to 100, were calculated by dividing the number of plaque-containing surfaces by the total number of available surfaces. A lower PCR score indicated better ability to control plaque. The difference in PCR scores between baseline and re-evaluation was used to assess OHS improvement in the participants.

Participants in the intervention group were divided into three subgroups according to their frequency of OSCA use to investigate the impact of frequency of use on the effectiveness of the intervention. Patients who used the OSCA at least four times a week on average and those who operated it less than once a week were classified as "highfrequency users" and "low-frequency users", respectively. All others were classified as "moderate-frequency users".

The measure of the functional preferences, discussed in previous study [10], comprised 13 items using a seven-point Likert scale (ranging from "strongly disagree" to "strongly agree"): 4 items focused on capability establishment, another 4 focused on opportunity creation, and the remaining 5 focused on motivation enhancement (Table 1). Composite variables were constructed with averaging of these items to assess the designed functions and resulted in Cronbach's $\alpha$ values of 0.850 for establishing capability, 0.782 for creating opportunity, and 0.818 for enhancing motivation. The overall likeability of the OSCA was assessed by 5 items using a seven-point Likert scale that were adapted from Hoj et al. [16]. A composite variable was also constructed with these 5 items and provided an assessment of the extent to which respondents liked the OSCA according to their experience (Cronbach's $\alpha=0.892$ ).

\subsection{Statistical Analysis}

Data were analyzed using SPSS (Version 20.0; SPSS Taiwan). Descriptive statistics were calculated for demographic characteristics, frequency of OSCA use, OHS, as well as functional preferences and overall likeability of the OSCA. The Shapiro-Wilk test was used to confirm the normality of oral hygiene index and the overall likeability of the OSCA. The difference in OHS between the two groups at baseline was analyzed using an independent samples $t$-test when normality was confirmed and using the Mann-Whitney $U$ test in other cases. Differences in OHS between baseline and re-evaluation for both 
groups were analyzed using paired $t$-tests when normality was confirmed and using the Wilcoxon signed-rank test in other cases. One-way ANOVA was used to compare intervention effectiveness between different levels of OSCA use frequency when normality was confirmed and using the Kruskal-Wallis test in other cases. Finally, multiple linear regression analysis was used to identify the factors associated with the effectiveness of the OSCA intervention as well as the relationship between functional preferences and the overall likeability of OSCA.

Table 1. Questions for functional preferences and overall likeability of the OCSA.

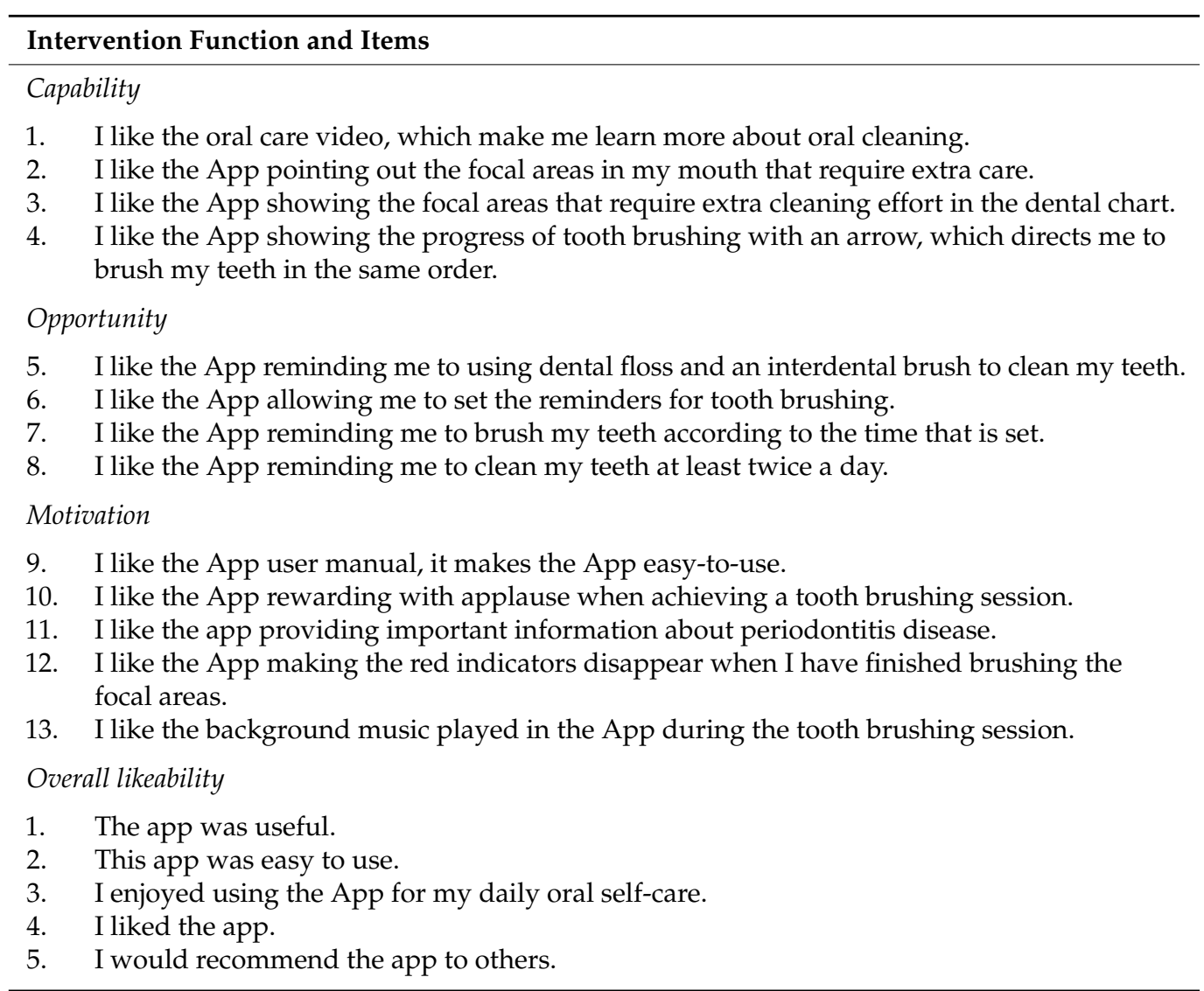

\section{Results}

\subsection{Participants and the Demographic}

Out of 150 participants, 39 participants in the control group underwent re-evaluation of their OHS, whereas 49 in the intervention group did and completed a questionnaire to measure the functional preferences and overall likeability of the OSCA.

The demographic characteristics of the participants are presented in Table 2. Of the 88 respondents, 45 (51.5\%) were males and 21 (23.9\%) were smokers. Participants ranged in age from 26 to 77 years, with a mean of $53.6 \pm 8.9$ years. Most respondents were between 45 and 64 years old, with $45.4 \%(n=40)$ reporting they were between 55 and 64 years old and $36.4 \%(n=32)$ reporting they were between 45 and 54 years old. Level of education varied from less than a high school education to a master's or PhD. degree, and most respondents reported attending college or university up to a bachelor's degree. Differences in age (independent samples $t$-test, $p=0.752)$ and education level ( $\chi^{2}$ analysis, $\left.p=0.624\right)$ between the two groups were not statistically significant. 
Table 2. Demographic characteristics of the study participants.

\begin{tabular}{|c|c|c|c|}
\hline & $\begin{array}{c}\text { Control Group } \\
\text { n (\%) }\end{array}$ & $\begin{array}{c}\text { Intervention Group } \\
\text { n (\%) }\end{array}$ & $\begin{array}{l}\text { Total } \\
\text { n (\%) }\end{array}$ \\
\hline $\mathrm{n}$ & $39(44.3)$ & $49(55.7)$ & $88(100)$ \\
\hline \multicolumn{4}{|l|}{ Gender } \\
\hline Male & $18(46.2)$ & $27(55.1)$ & $45(51.5)$ \\
\hline Female & $21(53.8)$ & $22(44.9)$ & $43(48.9)$ \\
\hline \multicolumn{4}{|l|}{ Age } \\
\hline$<35$ & $2(5.1)$ & $0(0.0)$ & $2(2.3)$ \\
\hline $35-44$ & $4(10.3)$ & $4(8.2)$ & $8(9.1)$ \\
\hline $45-54$ & $11(28.2)$ & $21(42.9)$ & $32(36.4)$ \\
\hline $55-64$ & $19(48.7)$ & $21(42.9)$ & $40(45.4)$ \\
\hline 65 or older & $3(7.7)$ & $3(6.1)$ & $6(6.8)$ \\
\hline \multicolumn{4}{|l|}{ Education } \\
\hline Less than high school & $9(23.1)$ & $7(14.3)$ & $16(18.2)$ \\
\hline High school & $11(29.2)$ & $13(26.5)$ & $24(27.3)$ \\
\hline College or university up to bachelor & $18(46.2)$ & $26(53.1)$ & $44(50.0)$ \\
\hline College or university up to master or PhD. & $1(2.6)$ & $3(6.1)$ & $4(4.5)$ \\
\hline \multicolumn{4}{|l|}{ Smoking } \\
\hline Yes & $9(23.1)$ & $12(24.5)$ & $21(23.9)$ \\
\hline No & $30(76.9)$ & $37(75.5)$ & $67(76.1)$ \\
\hline
\end{tabular}

\section{2. $\mathrm{OHS}$ and $\mathrm{OHBS}$}

The OHS of all study participants as measured by PCR scores at baseline and that at re-evaluation are presented in Table 3. At baseline, there was no significant difference in PCR scores between control and intervention groups after independent-samples $t$-test $(p=0.434)$. The significant difference in the improvement of OHS between the two groups was not detected by independent samples $t$-test $(p=0.06)$. However, OHS improvement significantly differed between low-, moderate-, and high-frequency users in one-way ANOVA (Figure 3), with the OHS improvement in the low-frequency users being significantly less than that in the moderate- and high-frequency users. Respondents' reported OHBs at baseline and re-evaluation are illustrated in Table 4. OHBs changes in control group and intervention group improved significantly according to Wilcoxon's signed-rank test $p=0.007$ and $p<0.001$, respectively). For the intervention group, a relationship between level of OSCA use frequency and reported change in OHBs was observed by the Kruskal-Wallis test $(p=0.002)$.

\subsection{Overall Likeability and Functional Preferences of the OSCA}

For the intervention group, the overall likeability of the OSCA was assessed through five items, and the normality of the composite variable of likeability was confirmed using the Shapiro-Wilk test $(p=0.081)$. The overall likeability and functional preferences of the OSCA for the different level of frequency users are presented in Table 5. The mean score of overall likeability for respondents in the intervention group was 5.85 , with a standard deviation of 0.76 , and the mean scores for the high-, moderate-, and low-frequency users were $6.36,6.14$, and 4.89 , respectively. The results of investigating functional preferences for establishing capability, creating opportunity, and enhancing motivation indicated that the low-frequency users gave the functional preferences a significantly lower rating compared with the moderate- and high-frequency users after Kruskal-Wallis test for independent samples. 
Table 3. OHS data for both groups at baseline and re-evaluation.

\begin{tabular}{cccc}
\hline & $\begin{array}{c}\text { Control Group } \\
\text { (Mean } \pm \text { SD) }\end{array}$ & $\begin{array}{c}\text { Intervention Group } \\
\text { (Mean } \pm \text { SD) }\end{array}$ & $\begin{array}{c}\text { Significance } \\
(\boldsymbol{p} \text {-Value })\end{array}$ \\
\hline Baseline & $72.38 \pm 15.59$ & $75.28 \pm 18.31$ & $0.223^{\mathrm{a}}$ \\
Re-evaluation & $54.59 \pm 16.30$ & $49.10 \pm 21.00$ & $0.091^{\mathrm{a}}$ \\
OHS improvement & $17.80 \pm 18.84$ & $26.17 \pm 21.76$ & $0.060^{\mathrm{b}}$ \\
\hline
\end{tabular}

a. Mann-Whitney $U$ test; $^{\text {b. Independent Samples } t \text {-test }}$

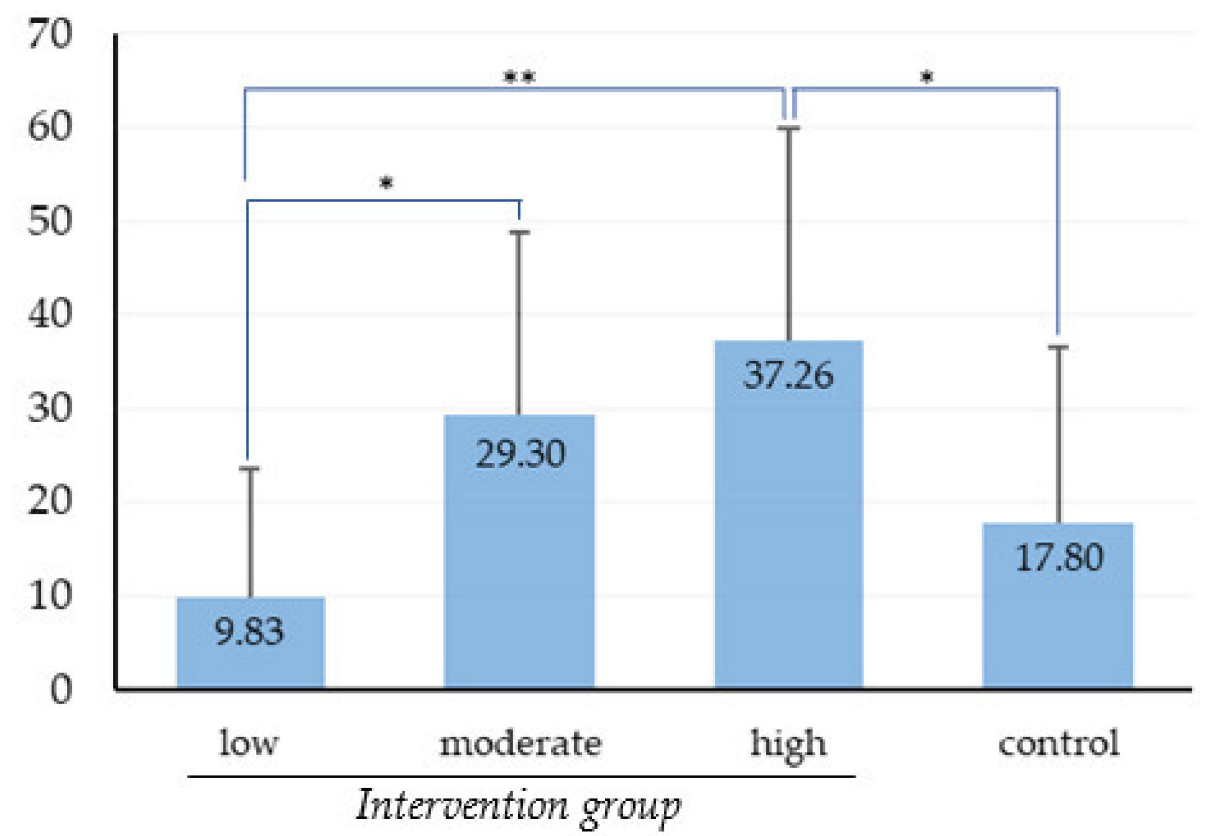

Figure 3. Comparison of OHS improvement between the control group and intervention group based on level of OSCA use frequency. Data are expressed as mean $\pm \mathrm{SD} .{ }^{*} p<0.05$ and ${ }^{* *} p<0.01$, one-way ANOVA and Scheffe test.

Table 4. OHBs data for both groups at baseline and re-evaluation.

\begin{tabular}{|c|c|c|c|c|}
\hline & \multicolumn{2}{|c|}{$\begin{array}{l}\text { Control Group } \\
n(\%)\end{array}$} & \multicolumn{2}{|c|}{$\begin{array}{c}\text { Intervention Group } \\
\mathrm{n}(\%)\end{array}$} \\
\hline & Baseline & Re-Evaluation & Baseline & Re-Evaluation \\
\hline \multicolumn{5}{|l|}{ Frequency of toothbrushing } \\
\hline Not every day & $1(2.6)$ & $1(2.6)$ & $2(4.1)$ & $0(0.0)$ \\
\hline Once a day & $11(28.2)$ & $9(23.1)$ & $11(22.4)$ & 7 (14.3) \\
\hline Twice a day or More than twice a day & $27(69.2)$ & $29(74.4)$ & $36(73.5)$ & $42(85.7)$ \\
\hline \multicolumn{5}{|l|}{ Duration of toothbrushing } \\
\hline Shorter than $1 \mathrm{~min}$ & $2(5.1)$ & $1(2.6)$ & $2(4.1)$ & $1(2.0)$ \\
\hline Longer than $3 \mathrm{~min}$ or $1 \mathrm{~min}$ & $22(56.4)$ & $24(61.5)$ & $32(65.3)$ & $15(30.7)$ \\
\hline $2 \min$ or $3 \mathrm{~min}$ & $15(38.5)$ & $14(35.9)$ & $15(30.6)$ & $33(67.3)$ \\
\hline \multicolumn{5}{|l|}{ Interdental cleaning } \\
\hline Rarely or Never & $8(20.5)$ & $2(5.2)$ & $18(36.7)$ & $6(12.2)$ \\
\hline A few times a week & $25(64.1)$ & $30(76.9)$ & $23(46.9)$ & $32(65.3)$ \\
\hline At least once a day & $6(15.4)$ & $7(17.9)$ & $8(16.4)$ & $11(22.4)$ \\
\hline Tongue cleaning & & & & \\
\hline Rarely or Never & $10(25.6)$ & $6(15.4)$ & $12(24.5)$ & $2(4.1)$ \\
\hline A few times a week & $20(51.3)$ & $24(61.5)$ & $29(59.2)$ & $35(71.4)$ \\
\hline At least once a day & $9(23.1)$ & $9(23.1)$ & $8(16.3)$ & $12(24.5)$ \\
\hline
\end{tabular}


Table 5. Overall likeability and functional preferences of the OSCA for participants in the intervention group.

\begin{tabular}{cccccc}
\hline & $n$ & $\begin{array}{c}\text { Likeability } \\
\text { Mean } \pm \text { SD) }\end{array}$ & $\begin{array}{c}\text { Capability } \\
\text { (Mean } \pm \text { SD) }\end{array}$ & $\begin{array}{c}\text { Opportunity } \\
\text { (Mean } \pm \text { SD) }\end{array}$ & $\begin{array}{c}\text { Motivation } \\
(\text { Mean } \pm \text { SD) }\end{array}$ \\
\hline Total & 49 & $5.85 \pm 0.76$ & $6.00 \pm 0.71$ & $5.77 \pm 0.70$ & $5.71 \pm 0.73$ \\
\hline High-level & 15 & \multicolumn{2}{c}{ Frequemncy of OSCA use } & & \\
Moderate-level & 20 & $6.36 \pm 0.43$ & $6.42 \pm 0.28$ & $6.00 \pm 0.57$ & $6.13 \pm 0.42$ \\
Low-level & 14 & $6.14 \pm 0.47$ & $6.31 \pm 0.49$ & $6.09 \pm 0.50$ & $6.03 \pm 0.39$ \\
\hline & $p$-value & $4.89 \pm 0.43$ & $5.11 \pm 0.46$ & $5.07 \pm 0.60$ & $4.81 \pm 0.58$ \\
\hline
\end{tabular}

\subsection{Regression Analysis}

OSCA use frequency $(p=0.035)$, change in OHBs $(p=0.006)$, and smoking $(p=0.017)$ significantly affected OHS improvement (Table 6). Except for the functional preference of creating opportunity, respondents reported that their preferences in establishing capability and enhancing motivation positively impacted on the overall likeability (Table 7). Level of OSCA use frequency was positively correlated with the overall likeability (Pearson's $r=0.745 ; p<0.001$ ) and change in OHBs (Pearson's $r=0.468 ; p=0.001$ ).

Table 6. Multiple regression of the improvement in OHS.

\begin{tabular}{cccc}
\hline Variable & Coefficient (Standard Error) & $\boldsymbol{t}$ & $\boldsymbol{p}$-Value \\
\hline Change of OHBs & $7.250(2.477)$ & 2.927 & 0.006 \\
Smoking & $-14.770(5.928)$ & -2.491 & 0.017 \\
Level of OSCA use frequency & $8.211(3.764)$ & 2.181 & 0.035 \\
Gender & $-5.594(4.981)$ & -1.123 & 0.268 \\
Age & $-5.526(3.523)$ & -1.568 & 0.124 \\
Education & $0.184(3.072)$ & 0.060 & 0.952 \\
\hline
\end{tabular}

Table 7. Multiple regression of the overall likeability of OSCA on the functional preferences.

\begin{tabular}{cccc}
\hline Variable & Coefficient (Standard Error) & $\boldsymbol{t}$ & $\boldsymbol{p}$-Value \\
\hline Establishing capability & $0.572(0.178)$ & 3.209 & 0.002 \\
Enhancing motivation & $0.429(0.182)$ & 2.269 & 0.028 \\
Creating opportunity & $-0.164(0.189)$ & -0.902 & 0.372 \\
\hline
\end{tabular}

\section{Discussion}

We have previously discussed the developmental theoretical framework and usability of the OSCA [10]. Objectives of this study were (1) to investigate the effectiveness of the OSCA intervention in improving OHS and facilitating change in OHBs and (2) to identify the factors that influence OHS improvement. The findings indicate that the effectiveness of the OSCA intervention is substantial.

OHS improvement was evaluated by the difference in PCR scores at baseline and re-evaluation. The participants in the intervention group demonstrated slightly better OHS improvement compared with the participants in the control group, and the low-frequency users in the intervention group demonstrated much less OHS improvement compared with the moderate- and high-frequency users (Figure 3). From the result, we can conclude that the intervention effectiveness was significant and positively correlated with the frequency of OSCA use. That is, participants could effectively improve their OHS by adhering to the OHIs with the OSCA and that frequency of app use affected the effectiveness of the OSCA intervention. It may be the case that frequent use of OSCA brought sufficient opportunities for impact on improving oral hygiene. Similar findings that adherence to OHIs is the main factor affecting OHS improvement for patients with periodontitis $[9,10]$ and that higher frequency of app use increases its effectiveness [16] have been reported. 
Furthermore, the effectiveness of the OSCA intervention was significantly affected not only by the frequency of its use but also by change in OHBs and smoking. Change in OHBs toward the target behavior of oral self-care had a positive impact on the improvement in the level of plaque control, indicating that better OHBs resulted in improved OHS, which is consistent with the findings of previous studies [10,28,29]. Research has found that good oral care plays an important role in maintaining oral health [4-7] and is the foundation for the long-term success of periodontal treatment [28-30]. Therefore, changing OHBs toward well performing daily oral self-care can improve OHS and enhance the treatment effect for periodontitis. Moreover, studies have shown that apps designed based on behavioral change theories are more effective at eliciting behavioral change in users $[13,20,31-34]$ and that personal information as well as evidence-based interventions can significantly help patients manage their conditions better [34,35]. In this study, OSCA was design to provide the intervention function of creating opportunity to the participants to well perform daily oral self-care. In addition, this study found that smoking significantly affected the effectiveness of plaque level improvement, which is similar to previously reported data [29,30,35]. Smoking is considered a risk factor in periodontal disease $[29,35,36]$ and has been proven to significantly affect the effectiveness of nonsurgical periodontal treatments $[5,35]$.

A relationship between level of OSCA use frequency and reported change in OHBs was observed. The low-frequency users exhibited significantly less change in OHBs compared with the moderate- and high-frequency users-that is, higher frequency of use was more likely to positively influence change in OHBs. Hoj et al. [16] applied a behavioral change theory to explore the mechanism of change related to physical activity behavior and found that frequent use of apps and behavioral change in physical activity were positively correlated. In this study, the moderate- and high-frequency users looked over the tailored information and performed oral self-care under the instruction of the OSCA more regularly and consequently achieved better OHBs.

A systematic review of Internet-based interventions to promote behavioral change found that intervention tools incorporating more behavioral change techniques performed better than did those employing fewer techniques $[37,38]$. In this study, the BCW framework [23] was adopted in the design of OSCA to provide the functions of establishing capability, enhancing motivation, and creating opportunity. Capability establishment with personalized, tailored information was designed to enhance the participants' knowledge of and skills in oral self-care. Motivation enhancement and opportunity creation were designed to increase engagement in achieving good daily oral hygiene.

Increasing frequency of use is an interesting topic for researchers and practitioners in the field of mobile health design [15]. In this study, the overall likeability of OSCA was given high ratings and was strongly positive correlated with frequency of use. That is, a higher perceived overall likeability may lead to more frequent OSCA use. This outcome could be attributable to the fact that the OSCA was designed with consideration of experts' opinions on practical guidance and evidence-based clinical practice to provide personalized intervention to the participants to establish capability for oral self-care, which was perceived acceptable and useful. The functions of motivation enhancement and opportunity creation were incorporated to increase frequency of OSCA use as well. This finding was consistent with previous reviews that apps with incorporating opinions of medical professionals were reported more likely to be perceived as having better feasibility and acceptability [13]. In addition, education, personalization and acceptable of app use were considered as key features for sustain use and the facilitation of behavior change [19,39].

In the intervention group, there were about one fifth low-frequency user, who rarely used the OSCA for their daily oral self-care. The exact reason for those participants not engaging with the app was not investigated in this study. Whatever the reason, it may be of interest to practitioners and developers who wish to use app-based intervention to promote health.

To minimize confounding factors, the participant's clinical examinations at baseline and re-evaluation were conducted by the same periodontist. In addition, identical oral 
hygiene instructions, including duration of toothbrushing (about 2 to $3 \mathrm{~min}$ ), brushing frequency (at least twice a day), and frequency of interdental cleaning (once a day), were delivered to the participants in the two group. Some clinical studies have shown that brushing for 2 or $3 \mathrm{~min}$ and twice a day was sufficient to maintain periodontal health in patients $[25,27,34]$. Therefore, we gave the oral hygiene instructions to the participants accordingly.

The primary strength of this study is that we validated the effectiveness of an appbased mobile intervention for precision oral self-care in patients with periodontitis. The app was designed to support changes in participants' OHBs and improve their oral hygiene by providing personalized and tailored information. In addition, the key factors that influenced the effectiveness of the OSCA intervention were also investigated.

There are two major limitations in this study that could be addressed in future research. First, the study focused on the short-term effectiveness of OSCA use from periodontal initial therapy to the re-evaluation (about 4-8 weeks). However, the re-evaluation is not the endpoint for the success of the periodontal therapy [40]. Studies have reported that the success rate of periodontitis therapy is less than $40 \%$ [41]. Further research is thus needed to confirm the long-term effectiveness of the app-based intervention for patients' oral self-care, especially the impact on the success of periodontal therapy. Second, participants' oral health, including PCR index, tooth type, bleeding on probing, and periodontal probing depth, was examined. In this study, the participant's oral hygiene status was evaluated only by the PCR score, which is one of the key indicators for assessing patients' ability for oral self-care [27]. The app-based intervention, in this study, may have had an impact on the other measures of oral health. Perhaps a future study could examine the intervention effectiveness with consideration of more indicators of oral health.

\section{Conclusions}

This study investigated the app-based intervention effectiveness for precision oral selfcare in periodontitis patients. We found that the intervention effectiveness in improving OHS was significant and strongly affected by the frequency of app use, change in OHBs, and smoking. Overall, our findings underscore the value of mobile apps for precision oral self-care and the need to further explore their long-term application and effectiveness in treating patients with periodontitis.

Author Contributions: Conceptualization, W.-J.C., Y.-L.W., Y.-H.C. and S.-Y.L.; data curation, W.-J.C., Y.-L.W. and S.-Y.L.; formal analysis, W.-J.C., Y.-L.W. and S.-Y.L.; investigation, W.-J.C., Y.-L.W., Y.-H.C. and S.-Y.L.; methodology, W.-J.C., Y.-L.W., Y.-H.C. and S.-Y.L.; project administration, W.-J.C. and Y.-H.C.; resources, W.-J.C.; software, W.-J.C.; supervision, W.-J.C.; validation, W.-J.C., Y.-L.W. and S.-Y.L.; writing—original draft, W.-J.C.; writing-review and editing, W.-J.C., Y.-L.W., Y.-H.C. and S.-Y.L. All authors have read and agreed to the published version of the manuscript.

Funding: This research received no external funding.

Institutional Review Board Statement: The study was conducted according to the guidelines of the Declaration of Helsinki, and approved by the Institutional Review Board of Chang Gung Memorial Hospital (Taoyuan, Taiwan) (Approval No. 201701853B0).

Informed Consent Statement: Informed consent was obtained from all subjects involved in the study.

Data Availability Statement: The datasets used and/or analyzed during the current study are available online.

Acknowledgments: The authors are grateful to the staff of Department of Periodontics, Chang Gung Memorial Hospital, Taoyuan, Taiwan. This research was supported by grant MOST 107-2221-E-182057 and MOST 109-2221-E-182-035 from the Ministry of Science and Technology, Taiwan.

Conflicts of Interest: The authors declare no conflict of interest. 


\section{References}

1. Geneva: World Health Organization. Oral Health. Available online: https://www.who.int/health-topics/oral-health\#tab=tab_1 (accessed on 12 January 2021).

2. Jin, L.J.; Lamster, I.B.; Greenspan, J.S.; Pitts, N.B.; Scully, C.; Warnakulasuriya, S. Global burden of oral diseases: Emerging concepts, management and interplay with systemic health. Oral. Dis. 2016, 22, 609-619. [CrossRef] [PubMed]

3. Geneva: World Health Organization. Oral health. Available online: https://www.who.int/bulletin/volumes/93/9/15-020915 /en/ (accessed on 15 April 2021).

4. Tonetti, M.S.; Jepsen, S.; Jin, L.; Otomo-Corgel, J. Impact of the global burden of periodontal diseases on health, nutrition and wellbeing of mankind: A call for global action. J. Clin. Periodontol. 2017, 44, 456-462. [CrossRef] [PubMed]

5. Tiffany, B.; Blasi, P.; Catz, S.; McClure, J.B. Mobile apps for oral health promotion: Content review and heuristic usability analysis. JMIR Mhealth Uhealth 2018, 6, e11432. [CrossRef]

6. Carra, M.C.; Detzen, L.; Kitzmann, J.; Woelber, J.P.; Ramseier, C.A.; Bouchard, P. Promoting behavioural changes to improve oral hygiene in patients with periodontal diseases: A systematic review. J. Clin. Periodontol. 2020, 47 (Suppl. 22), 72-89. [CrossRef]

7. Misra, S.; Daly, B.; Dunne, S.; Millar, B.; Packer, M.; Asimakopoulou, K. Dentist-patient communication: What do patients and dentists remember following a consultation? Implications for patient compliance. Patient Prefer. Adherence 2013, 7, 543-549.

8. Newton, J.T.; Asimakopoulou, K. Minimally invasive dentistry: Enhancing oral health-related behavior through behavior change techniques. Br. Dent. J. 2017, 223, 147-150. [CrossRef]

9. Johnston, M. Improving the reporting of behaviour change interventions. Eur. Health Psychol. 2016, 16, 181-189.

10. Chang, W.J.; Lo, S.Y.; Kuo, C.L.; Wang, Y.L.; Hsiao, H.C. Development of an intervention tool for precision oral self-care: Personalized and evidence-based practice for patients with periodontal disease. PLoS ONE 2019, 14, e0225453. [CrossRef] [PubMed]

11. Renz, A.; Ide, M.; Newton, T.; Robinson, P.G.; Smith, D. Psychological interventions to improve adherence to oral hygiene instructions in adults with periodontal diseases. Cochrane Database Syst. Rev. 2007, 2, CD005497.

12. Milne-Ives, M.; Lam, C.; Van Velthoven, M.H.; Meinert, E. Mobile apps for health behavior change: Protocol for a systematic review. JMIR Res. Protoc. 2020, 9, e16931. [CrossRef]

13. Fitzgerald, M.; McClelland, T. What makes a mobile app successful in supporting health behaviour change? Health Educ. J. 2017, 76, 373-381. [CrossRef]

14. Research2Guidance. 325,000 Mobile Health Apps Available in 2017-Android Now the Leading mHealth Platform. Available online: https:/ / research2guidance.com/325000-mobile-health-apps-available-in-2017/ (accessed on 12 January 2021).

15. Lopez, K.D.; Chae, S.; Michele, G.; Fraczkowski, D.; Habibi, P.; Chattopadhyay, D.; Donevant, S.B. Improved readability and functions needed for mHealth apps targeting patients with heart failure: An app store review. Res. Nurs. Health 2020, 44, 71-80. [CrossRef] [PubMed]

16. Hoj, T.H.; Covey, E.L.; Jones, A.C.; Haines, A.C.; Hall, P.C.; Crookston, B.T.; West, J.H. How do apps work? An analysis of physical activity app users' perceptions of behavior change mechanisms. JMIR Mhealth Uhealth 2017, 5, e114. [CrossRef] [PubMed]

17. Villinger, K.; Wahl, D.R.; Boeing, H.; Schupp, H.T.; Renner, B. The effectiveness of app-based mobile interventions on nutrition behaviours and nutrition-related health outcomes: A systematic review and meta-analysis. Obes. Rev. 2019, 20, 1465-1484. [CrossRef]

18. Wilhide, C.; Peeples, M.; Kouyate, R. Evidence-based mHealth chronic disease mobile app intervention design: Development of a framework. JMIR Res. Protoc. 2016, 5, e25. [CrossRef]

19. Payne, H.E.; Lister, C.; West, J.H.; Bernhardt, J.M. Behavioral functionality of mobile apps in health interventions: A systematic review of the literature. JMIR Mhealth Uhealth 2015, 3, e20. [CrossRef]

20. Direito, A.; Dale, L.; Shields, E.; Dobson, R.; Whittaker, R.; Maddison, R. Do physical activity and dietary smartphone applications incorporate evidence-based behaviour change techniques? BMC Public Health 2014, 14, 646. [CrossRef] [PubMed]

21. Parker, K.; Bharmal, R.V.; Sharif, M.O. The availability and characteristics of patient-focused oral hygiene apps. Br. Dent. J. 2019, 226, 600-604. [CrossRef]

22. Gordon, W.J.; Landman, A.; Zhang, H.; Bates, D.W. Beyond validation: Getting health apps into clinical practice. NPJ Digit. Med. 2020, 3, 14. [CrossRef]

23. Michie, S.; van Stralen, M.; West, R. The behaviour change wheel: A new method for characterising and designing behaviour change interventions. Implement. Sci. 2011, 6, 42. [CrossRef]

24. Segelnick, S.L.; Weinberg, M.A. Reevaluation of initial therapy: When is the appropriate time? J. Periodontol. 2006, 77, 1598-1601. [CrossRef]

25. Brein, D.J.; Fleenor, T.J.; Kim, S.W.; Krupat, E. Using the theory of planned behavior to identify predictors of oral hygiene: A collection of unique behaviors. J. Periodontol. 2016, 87, 312-319. [CrossRef] [PubMed]

26. O'Leary, T.J.; Drake, R.B.; Naylor, J.E. The plaque control record. J. Periodontol. 1972, 43, 38. [CrossRef] [PubMed]

27. Park, S.H.; Cho, S.H.; Han, J.Y. Effective professional intraoral tooth brushing instruction using the modified plaque score: A randomized clinical trial. J. Periodontal Implant Sci. 2018, 48, 22-33. [CrossRef] [PubMed]

28. Klein, M.; Mogles, N.; Van Wissen, A. Intelligent mobile support for therapy adherence and behavior change. J. Biomed. Inform. 2014, 51, 137-151. [CrossRef] [PubMed] 
29. Tang, M.Y.; Smith, D.M.; Mc Sharry, J.; Hann, M.; French, D.P. Behavior change techniques associated with changes in postintervention and maintained changes in self-efficacy for physical activity: A systematic review with meta-analysis. Ann. Behav. Med. 2019, 53, 801-815. [CrossRef] [PubMed]

30. Jeon, E.; Park, H.A. Development of the IMB model and an evidence-based diabetes self-management mobile application. Healthc. Inform. Res. 2018, 24, 125-138. [CrossRef]

31. Wensing, M.; Huntink, E.; van Lieshout, J.; Godycki-Cwirko, M.; Kowalczyk, A.; Jager, C.; Steinhäuser, J.; Aakhus, E.; Flottorp, S.; Eccles, M.; et al. Tailored implementation of evidence-based practice for patients with chronic diseases. PLoS ONE 2014, 9, 101981. [CrossRef]

32. Shamani, S.; Jansson, L. Oral hygiene behaviour change during the nonsurgical periodontal treatment phase. Open Dent. J. 2012, 6, 190-196. [CrossRef]

33. Arweiler, N.B.; Auschill, T.M.; Sculean, A. Patient self-care of periodontal pocket infections. Periodontol. 2000 2018, 76, 164-179. [CrossRef]

34. Wilder, R.S.; Bray, K.S. Improving periodontal outcomes: Merging clinical and behavioral science. Periodontol. 2000 2016, 71, 65-81. [CrossRef]

35. Kumar, S. Evidence-Based Update on Diagnosis and Management of Gingivitis and Periodontitis. Dent. Clin. N. Am. 2019, 63, 69-81. [CrossRef]

36. Nazir, M.A. Prevalence of periodontal disease, its association with systemic diseases and prevention. Int. J. Health Sci. 2017, 11, 72-80.

37. Webb, T.; Joseph, J.; Yardly, L.; Michie, S. Using the internet to promote health behavior change: A systematic review and meta-analysis of the impact of theoretical basis, use of behavior change techniques, and mode of delivery on efficacy. J. Med. Internet Res. 2010, 12, 4. [CrossRef]

38. Lewis, T.L.; Wyatt, J.C. App usage factor: A simple metric to compare the population impact of mobile medical apps. J. Med. Internet Res. 2015, 17, 200. [CrossRef]

39. Holzinger, A. Interactive machine learning for health informatics: When do we need the human-in-the-loop? Brain Inform. 2016, 3, 119-131. [CrossRef] [PubMed]

40. Loos, B.G.; Needleman, I. Endpoints of active periodontal therapy. J. Clin. Periodontol. 2020, 47, 61-71. [CrossRef] [PubMed]

41. Van der Weijden, G.A.; Gijs, J.; Dekkers, G.J.; Slot, D.E. Success of non-surgical periodontal therapy in adult periodontitis patients: A retrospective analysis. Int. J. Dent. Hygiene. 2019, 17, 309-317. [CrossRef] [PubMed] 